\title{
Chest pain in the ambulance; prevalence, causes and outcome - a retrospective cohort study
}

\author{
Claus Kjær Pedersen ${ }^{1 *}$ DD, Carsten Stengaard ${ }^{1}$, Kristian Friesgaard ${ }^{2,3}$, Karen Kaae Dodt ${ }^{4}$, Hanne Maare Søndergaard ${ }^{5}$, \\ Christian Juhl Terkelsen ${ }^{1}$ and Morten Thingemann Bøtker ${ }^{2,3}$
}

\begin{abstract}
Background: Chest pain is common in acute ambulance transports. This study aims to characterize and compare ambulance-transported chest pain patients to non-chest pain patients and evaluate if patient characteristics and accompanying symptoms accessible at the time of emergency call can predict cause and outcome in chest pain patients.

Methods: Retrospective, observational population-based study, including acute ambulance transports. Patient characteristics and symptoms are included in a multivariable risk model to identify characteristics, associated with being discharged without an acute cardiac diagnosis and surviving 30 days after chest pain event.

Results: In total, 10,033 of 61,088 (16.4\%) acute ambulance transports were due to chest pain. In chest pain patients, 30-day mortality was $2.1 \%(95 \% \mathrm{Cl} 1.8-2.4)$ compared to $6.0 \%(95 \% \mathrm{Cl} 5.7-6.2)$ in non-chest pain patients. Of chest pain patients, 1054 (10.5\%) were diagnosed with acute myocardial infarction, and 5068 (50.5\%) were discharged without any diagnosis of disease. This no-diagnosis group had very low 30-day mortality, $0.4 \%$ (95\%Cl 0.2-0.9). Female gender, younger age, chronic pulmonary disease, absence of accompanying symptoms of dyspnoea, radiation, severe pain for $>5$ min, clammy skin, uncomfortable, and nausea were associated with being discharged without an acute cardiac diagnosis and surviving 30 days after a chest pain event.

Conclusion: Chest pain is a common reason for ambulance transport, but the majority of patients are discharged without a diagnosis and with a high survival rate. Early risk prediction seems to hold a potential for resource downgrading and thus cost-saving in selected chest pain patients.
\end{abstract}

Keywords: ACS, AMI, Chest pain, Prehospital diagnosis, Prehospital triage, EMS

\section{Introduction}

Chest pain is one of the most frequent symptoms among patients contacting the emergency medical services (EMS) and emergency departments (EDs) $[1,2]$. In the United States, the estimated number of ED contacts due to chest pain exceeded 7 million in 2015 [3, 4]. Health care expenses for handling these patients are massive and are mainly driven by length of hospital stay [5]. Depending on the underlying cause, an acute EMS-

\footnotetext{
* Correspondence: claus.kjaer.pedersen@clin.au.dk

${ }^{1}$ Department of Cardiology, Aarhus University Hospital, Palle Juul-Jensens Boulevard 99, 8200 Aarhus N, Denmark

Full list of author information is available at the end of the article
}

response and subsequent hospitalization may prove relevant or non-relevant in chest pain patients.

Since chest pain is associated with potential life threatening conditions like acute myocardial infarction (AMI), pulmonary embolism or acute aortic dissection, the EMS response is most often dispatched in an "all or nothing"fashion with a high triage level and immediate response. This level of triage is relevant in patients with a subsequent confirmed serious/adverse diagnosis and may favour an improved outcome [6, 7]. While patients with AMI often present with chest pain, the majority of patients presenting with chest pain may suffer from other more harmless conditions [8-10]. A mismatch like this could lead to overtriage and an excess of non-relevant

(c) The Author(s). 2019 Open Access This article is distributed under the terms of the Creative Commons Attribution 4.0 International License (http://creativecommons.org/licenses/by/4.0/), which permits unrestricted use, distribution, and reproduction in any medium, provided you give appropriate credit to the original author(s) and the source, provide a link to the Creative Commons license, and indicate if changes were made. The Creative Commons Public Domain Dedication waiver (http://creativecommons.org/publicdomain/zero/1.0/) applies to the data made available in this article, unless otherwise stated. 
EMS responses in chest pain patients. Secondarily this leads to high EMS-resource consumption, crowding, and prolonged length-of-stay (LOS) in EDs and coronary care units (CCUs) $[2,8,11]$.

Consequently, there is a need for evaluating prehospital over-triage and non-relevant EMS use in chest pain patients by examining diagnoses and mortality rates in patients presenting with chest pain and a need for addressing ways of early discrimination between high- and low-risk patients and thus support the decision of when an acute EMS response is relevant or may be downgraded. Structured use of patient characteristics and presence/absence of accompanying symptoms could be a key factor in improving the triage in chest pain patients [12]. Therefore, we aimed to compare EMS-patients according to presence of chest pain and to identify predictors of acute cardiac conditions and death.

\section{Methods}

Aim

The specific aims of the present study were, in patients transported to hospital by ambulance:

- To describe the prevalence of chest pain and compare patient characteristics, mortality rates, diagnostic patterns, LOS, and place of primary admittance between chest pain and non-chest pain patients

- To compare mortality rates and LOS among chest pain patients according to the final diagnoses

- To identify predictors (available at the time of ambulance dispatch) of "potential for downgrading EMS response" in chest pain patients, indicated by discharge without an acute cardiac diagnosis and survival for more than 30 days after chest pain event.

\section{Study design and setting}

This retrospective, observational population-based study was approved by The Danish Data Protection Agency, Central Denmark Region (ref. number 1-16-02-788-17) and the Danish Patient Safety Authority (ref. number 33013-2321).

The Danish health care system, including the EMS system, has previously been described [13, 14]. In Denmark EMS assistance can be requested either via medical emergency call (1-1-2) or by request from a general practitioner (GP) both during day-time and out-ofhours. When EMS is requested via 1-1-2, the medical emergency/injury is triaged according to the symptom/ criteria-based Danish Index for Emergency Care [15]. However, when ambulance dispatch is requested by a GP, the prehospital response is based on the GPs' clinical assessment rather than dispatch criteria [14].

\section{Data collection and processing}

We collected dispatch information and information on the preceding 1-1-2 call and merged these with data from the Danish National Patient Registry to include data on hospital admissions and existing comorbidity [16]. We acquired vital status from the public Danish Civil Registration System. The use of the Danish health registries in research has previously been validated $[17,18]$.

\section{Selection of participants}

This study included patients transported to hospital by ambulance in the Central Denmark region in a 1 -year period from May 1st 2015 to April 30th 2016. We included patients transported after 1-1-2 call and patients transported following request from GP. We excluded transports missing information on symptoms or index diagnosis and transports terminated before reaching hospital, see Additional files 1 and 2.

\section{Variables}

Way of entrance into the EMS system was categorized as "112-requested" or "GP-requested". Chest pain was identified through dispatch codes for patients undergoing criteria-based dispatch (112-requested) and through specific text searches in electronic prehospital patient files (in GP-requested transports). The automatic text search to identify chest pain/discomfort in the patient files was verified by manual review of 3500 patient files (5\%). Details on the automatic text search and validation are described in Additional file 3. We retrieved ICD-10 diagnoses given during index admission to identify final diagnosis of index admission, and within 10 years prior to the admission date to calculate Charlson Comorbidity Index (CCI), and to identify known comorbidity, see Additional file 4 [19].

We categorized final diagnosis according to the ICD10 codes assigned to the patient as primary diagnoses during the admission following the EMS transport (= index admission):

1) Acute cardiac conditions (AMI and other acute/ potentially life-threatening cardiovascular diagnoses)

2) Other diagnoses according to ICD-10 chapters

3) No final diagnosis (ICD-10 diagnosis of "Rxx.x", "Z03.x" or "Z04.x" AND none of the above mentioned during index admission)

See Additional file 4 for specific ICD-10 codes and categories and exceptions in category 3.

\section{Outcomes}

Primary outcome: Death within 30 days of admission. 
Secondary outcome: Acute cardiac diagnosis during index admission

Composite secondary endpoint: To investigate if the ambulance transport and the succeeding hospital admissions in chest pain patients were appropriate or not, we constructed a combined secondary outcome of being discharged without an acute cardiac condition and surviving more than 30 days after chest pain event.

See Additional file 4 for specific ICD 10 codes.

\section{Statistical methods}

Categorical data are presented as numbers and proportions. Continuous data are presented as means with $95 \%$ confidence interval $(\mathrm{CI})$ or medians and interquartile ranges (IQR). For comparison between groups, Fisher's exact test and Pearson's chi-squared test were used for categorical data, and unpaired samples T-test and Wilcoxon Rank Sum for continuous data as appropriate. Mortality is described as Kaplan-Meier curves truncated at 30 days. Follow-up was terminated November 14th 2016 and only first transport registered for each patient was included into mortality analysis, see flowchart in Additional file 1.

We performed two predefined multivariable prediction models, using binary regression analyses in patients with chest pain, to evaluate the association between the secondary composite endpoint of not being diagnosed with an acute cardiac condition and surviving more than 30 days and different patient characteristics and accompanying symptoms - information that is potentially available at the time of emergency call. The first model, applied to all patients suffering chest pain, included: 1) age, gender, prior AMI, diabetes mellitus, known congestive heart disease, chronic pulmonary disease, and moderate to severe renal disease. The second model included the same patient characteristics as in model 1 , but also accompanying symptoms. This was systematically registered only for those patients undergoing criteria-based dispatch (not GP requested dispatch) and thus, this model was applied to that sub-group only.

Age was included as age-groups $(<=29,30-49,50-59$, $60-69, \geq 70$ years). The mentioned predictor variables were included in multivariable analysis.

Regression models were evaluated 1) by plotting predicted and observed frequencies against age group as relevant in subgroups according to the remaining variables, 2) by likelihood-ratio test, and 3) by calculation of goodnessof-fit. All calculations were two-sided and $P$-values $<0.05$ were considered statistically significant. Analyses were performed using STATA Intercooled software, version 15.1.

\section{Results}

\section{Characteristics of study subjects}

We identified 71,891 acute ambulance transports in the prehospital dispatch system during the study period, see flowchart in Additional file 1. After exclusion of patients not transported to hospital and patients with no available description of symptoms and final diagnosis, we included 61,088 transports. We analysed mortality in 47, 601 unique first patient contacts. Patient and transport characteristics are summarized in Table 1.

\section{Main results}

Chest pain was the main symptom in 10,033 (16.4\%) of ambulance transports. This equals eight transports due to chest pain per 1000 inhabitants per year in the Central Denmark Region. Thirty-day mortality rate was considerably lower in chest pain patients than in patients without chest pain, $2.1 \%(95 \% \mathrm{CI} 1.8-2.4)$ compared to $6.0 \%$ (95\%CI 5.7-6.2), Fig. 1a. After exclusion of patients requesting an ambulance due to conditions with very high risk of mortality (unconsciousness, cardiac arrest, suspected death, drowning, diving accidents, foreign matter blocking airway and GP-triaged patients dying within first admission day), the 30-day mortality in nonchest pain patients were still more than twice as high as in chest pain patients $(4.7 \%(95 \% \mathrm{CI} 4.5-5.0)$ vs $2.1 \%$ (95\%CI 1.8-2.4)). Chest pain patients were more likely to receive a prehospital A-triage by the EMCC compared to non-chest pain patients ( $88 \%$ vs $38 \%$ ), they had a higher frequency of prior AMI (16\% vs $5 \%$ ), and were more often triaged directly to a department of cardiology ( $46 \%$ vs $7 \%$ ). Median LOS was more than twice as long in chest pain patients, compared to non-chest pain patients $(11.2$ vs $4.8 \mathrm{~h})(p<0.001$ for all mentioned variables), see Table 1 .

\section{Diagnostic patterns and outcome}

A disease-specific diagnosis was established in $49.5 \%$ of chest pain patients (AMI 10.5\%, other acute cardiovascular conditions $2.6 \%$, and other diagnoses $36.4 \%$ ) whereas $50.5 \%$ received no disease-specific diagnosis during index admission, Table 1 . The rate of acute cardiac diagnoses assigned during the index admission, was higher in patients with chest pain compared to patients without chest pain $(13 \%$ vs $2 \%)(p<0.001)$, see Table 1 . However, patients presenting with chest pain were also more often discharged without a disease-specific diagnosis $(51 \%$ vs $19 \%)(p<0.001)$. The 30 -day mortality in chest pain patients was lowest in those not receiving a disease specific diagnosis during index admission $(<0.5 \%)$, Table 2 and Fig. $1 b$.

\section{Potential for downgrading EMS response}

When including all chest pain patients into our multivariable model (Model 1), female gender, younger age, congestive heart disease, and chronic pulmonary disease were all associated with being discharged without an acute cardiac diagnosis. Age below 70 and no chronic 
Table 1 Baseline characteristics, prevalence of chest pain, incidence of AMI and other diagnoses among all GP- and 1-1-2 triaged patients

\begin{tabular}{|c|c|c|c|c|}
\hline & \multirow[t]{2}{*}{ All transports } & \multicolumn{2}{|l|}{ Chest pain } & \multirow[t]{2}{*}{$p$-value } \\
\hline & & No & Yes & \\
\hline Acute ambulance transports, n(\%) & $61,088(100.0 \%)$ & $51,055(83.6 \%)$ & $10,033(16.4 \%)$ & \\
\hline Registrations, N per 1000 inhabitants per year & 47.5 & 39.7 & 7.8 & \\
\hline \multicolumn{5}{|l|}{ Way of ambulance request, $\mathrm{n}(\%)$} \\
\hline GP & $32,036(52.4 \%)$ & $25,743(50.4 \%)$ & $6293(62.7 \%)$ & \multirow[t]{2}{*}{$<0.001$} \\
\hline 112 & $29,052(47.6 \%)$ & $25,312(49.6 \%)$ & $3740(37.3 \%)$ & \\
\hline \multicolumn{5}{|l|}{ Pre-hospital triage, n(\%) } \\
\hline A & $28,230(46.3 \%)$ & $19,420(38.1 \%)$ & $8810(87.8 \%)$ & \multirow[t]{3}{*}{$<0.001$} \\
\hline B & $24,754(40.6 \%)$ & $23,813(46.7 \%)$ & $941(9.4 \%)$ & \\
\hline C & $8046(13.2 \%)$ & $7766(15.2 \%)$ & $280(2.8 \%)$ & \\
\hline Male gender, n(\%) & $31,563(51.7 \%)$ & $26,005(50.9 \%)$ & $5558(55.4 \%)$ & $<0.001$ \\
\hline Age, median (IQR) & $65(45,78)$ & $65(42,78)$ & $66(52,77)$ & $<0.001$ \\
\hline Diabetes, n(\%) & $7080(11.6 \%)$ & $5754(11.3 \%)$ & $1326(13.2 \%)$ & $<0.001$ \\
\hline Prior AMI, n(\%) & $4273(7.0 \%)$ & $2718(5.3 \%)$ & 1555 (15.5\%) & $<0.001$ \\
\hline \multicolumn{5}{|l|}{ Charlson comorbidity index, n(\%) } \\
\hline 0 & $14,736(24.2 \%)$ & $13,158(25.8 \%)$ & $1578(15.7 \%)$ & \multirow[t]{4}{*}{$<0.001$} \\
\hline $1-2$ & $12,285(20.2 \%)$ & $9586(18.8 \%)$ & $2699(26.9 \%)$ & \\
\hline $3-4$ & $14,698(24.1 \%)$ & $11,994(23.6 \%)$ & $2704(27.0 \%)$ & \\
\hline$>=5$ & $19,224(31.5 \%)$ & $16,173(31.8 \%)$ & $3051(30.4 \%)$ & \\
\hline Length of stay, hours, median(IQR) & $5.8(2.0,37.3)$ & $4.8(1.9,36.0)$ & $11.2(3.6,42.7)$ & \multirow[t]{2}{*}{$<0.001$} \\
\hline Place of first admittance (Non-cardiac/CCU/Invasive centre) ${ }^{a}$ & $52,981 / 5267 / 2840$ & $47,613 / 2357 / 1085$ & $5368 / 2910 / 1755$ & \\
\hline \multicolumn{5}{|l|}{ Final diagnosis } \\
\hline Acute cardiac conditions, n(\%): & $2333(3.8 \%)$ & $1015(2.0 \%)$ & $1318(13.1 \%)$ & \multirow[t]{10}{*}{$<0.001$} \\
\hline Acute myocardial infarction: & $1482(2.4 \%)$ & $428(0.8 \%)$ & $1054(10.5 \%)$ & \\
\hline STEMI & $453(0.7 \%)$ & $113(0.2 \%)$ & $340(3.4 \%)$ & \\
\hline NSTEMI & $1029(1.7 \%)$ & $315(0.6 \%)$ & $714(7.1 \%)$ & \\
\hline Other acute cardiovascular conditions: & $851(1.4 \%)$ & $587(1.1 \%)$ & $264(2.6 \%)$ & \\
\hline UAP & $181(0.3 \%)$ & $41(0.1 \%)$ & $140(1.4 \%)$ & \\
\hline Cardiac arrest & $204(0.3 \%)$ & $193(0.4 \%)$ & $11(0.1 \%)$ & \\
\hline Ventricular tachycardia & $53(0.1 \%)$ & $38(0.1 \%)$ & $15(0.1 \%)$ & \\
\hline Aortic dissection & $114(0.2 \%)$ & $93(0.2 \%)$ & $21(0.2 \%)$ & \\
\hline Pulmonary Embolism & $299(0.5 \%)$ & $222(0.4 \%)$ & $77(0.8 \%)$ & \\
\hline Other cardiovascular and non-cardiovascular conditions, n(\%): & $44,125(72.2 \%)$ & $40,478(79.3 \%)$ & $3647(36.4 \%)$ & \multirow[t]{11}{*}{$<0.001$} \\
\hline Certain infectious and parasitic diseases & $1928(3.2 \%)$ & $1822(3.6 \%)$ & $106(1.1 \%)$ & \\
\hline Neoplasms & $580(0.9 \%)$ & $559(1.1 \%)$ & $21(0.2 \%)$ & \\
\hline Endocrine, nutritional and metabolic diseases & $1373(2.2 \%)$ & $1306(2.6 \%)$ & $67(0.7 \%)$ & \\
\hline Mental and behavioral disorders & $1959(3.2 \%)$ & $1851(3.6 \%)$ & $108(1.1 \%)$ & \\
\hline Diseases of the nervous system & $2004(3.3 \%)$ & $1962(3.8 \%)$ & $42(0.4 \%)$ & \\
\hline Diseases of the circulatory system (other than acute) & $6332(10.4 \%)$ & $4598(9.0 \%)$ & $1734(17.3 \%)$ & \\
\hline Diseases of the respiratory system & $6453(10.6 \%)$ & $5854(11.5 \%)$ & $599(6.0 \%)$ & \\
\hline Diseases of the digestive system & $3405(5.6 \%)$ & $3097(6.1 \%)$ & $308(3.1 \%)$ & \\
\hline Diseases of the musculoskeletal system and connective tissue & $1239(2.0 \%)$ & $1126(2.2 \%)$ & $113(1.1 \%)$ & \\
\hline Diseases of the genitourinary system & $1853(3.0 \%)$ & $1760(3.4 \%)$ & $93(0.9 \%)$ & \\
\hline
\end{tabular}


Table 1 Baseline characteristics, prevalence of chest pain, incidence of AMI and other diagnoses among all GP- and 1-1-2 triaged patients (Continued)

\begin{tabular}{|c|c|c|c|c|}
\hline & \multirow[t]{2}{*}{ All transports } & \multicolumn{2}{|l|}{ Chest pain } & \multirow[t]{2}{*}{$p$-value } \\
\hline & & No & Yes & \\
\hline Injury, poisoning and certain other consequences of external causes & $12,739(20.9 \%)$ & $12,587(24.7 \%)$ & $152(1.5 \%)$ & \\
\hline Other diagnoses $^{\mathrm{b}}$ & 4260 (7.0\%) & 3956 (7.7\%) & $304(3.0 \%)$ & \\
\hline No final diagnosis, $\mathrm{n}(\%)$ : & $14,630(23.9 \%)$ & $9562(18.7 \%)$ & $5068(50.5 \%)$ & $<0.001$ \\
\hline Symptoms, signs and abnormal clinical and laboratory findings & $8571(14.0 \%)$ & $6177(12.1 \%)$ & $2394(23.9 \%)$ & \\
\hline $\begin{array}{l}\text { Medical observation and evaluation for suspected diseases and } \\
\text { conditions or other reasons }\end{array}$ & $6059(9.9 \%)$ & $3385(6.6 \%)$ & $2674(26.7 \%)$ & \\
\hline
\end{tabular}

GP General practitioner, IQR interquartile range, UAP Unstable angina pectoris, STEMI ST-segment elevation myocardial infarction, NSTEMI non ST-segment elevation myocardial infarction

a Place of first admittance (Non-cardiac department, including ED / CCU /Invasive Centre)

${ }^{\mathrm{b}}$ Chapters containing $<1 \%$ of the total number of transports

pulmonary disease were all associated with surviving 30 days after a chest pain event, see Table 3.

When combining Model 1 with accompanying symptoms as predictors in the multivariable analysis (Model 2) in the subgroup of chest pain patients undergoing criteria-based dispatch, both absence of dyspnoea, absence of radiation/severe pain for $>5 \mathrm{~min}$ and absence of clammy skin/uncomfortable/nausea were associated with being discharged without a severe cardiac diagnosis. Absence of an accompanying symptom of non-responsiveness and dyspnoea were associated with surviving 30 days after a chest pain event, see Table 4.

Female gender, younger age, chronic pulmonary disease, absence of dyspnoea, absence of radiation/severe pain for $>5 \mathrm{~min}$ and absence of clammy skin/uncomfortable/nausea were associated with the combined endpoint of being discharged without an acute cardiac diagnosis and surviving 30 days after chest pain event.

When applied in a diagnostic model, Model 2 identified $14 \%$ of the patients eventually discharged without an acute cardiac diagnosis and surviving 30 days after a chest pain event, with a sensitivity of $99 \%$, a negative predictive value (NPV) of $98 \%$, and a negative likelihood-ratio of 0.08 . Thirty-day mortality in these patients was $0.7 \%$.

\section{Limitations}

This large study is based on all registrations of ambulance transports in the Central Denmark Region in a 1 year period, combined with diagnoses and vital status from validated national registers. However, we did exclude $6 \%$ of the cohort due to missing data on either symptom or diagnosis, and selection bias cannot be excluded. Available characteristics, diagnoses and outcome in these patients are presented in Additional file 2. In short, we see a very low incidence of AMI and other acute cardiac diagnoses $(<2 \%)$ in the symptom-missing patients and likewise a low incidence of chest pain (6\%) in the diagnose-missing patients. Thirty-day mortality in the symptom-missing group is not different from the
A symptoms and outcome in patients transported by ambulance
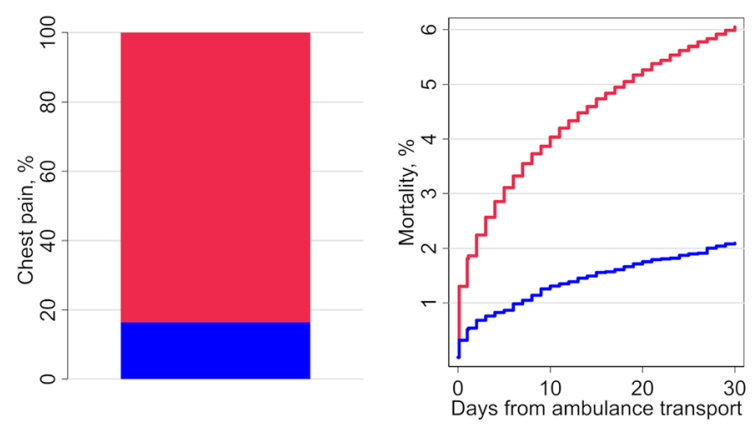

No chest pain

Chest pain
B Causes and mortality in chest pain patients
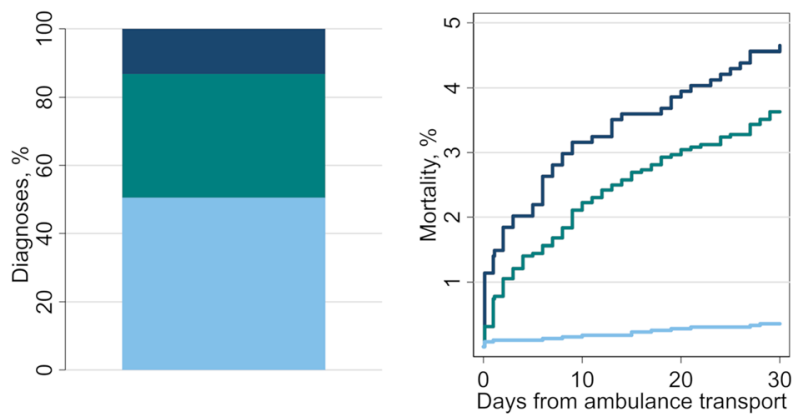

Acute cardiac diagnoses

Other diagnoses

No final diagnosis

Fig. 1 a Presence of chest pain and outcome in patients transported by ambulance. $\mathbf{b}$ Causes and outcome in chest pain patients divided in 3 groups: "Acute cardiac diagnoses", "Other diagnoses" and "No diagnosis". See Additional file 5 for raw values and categories (a) and time-toevent-or-censoring and censor status per patient (b) 
Table 2 Length of stay and 30-day mortality among GP- and 1-1-2 triaged chest pain patients according to ICD10-diagnosis

\begin{tabular}{|c|c|c|c|}
\hline & Total transports, $\mathrm{n}$ & $\begin{array}{l}\text { Median LOS, } \\
\text { hours }(\mathrm{Q} 1 ; \mathrm{Q} 3)\end{array}$ & $\begin{array}{l}\text { 30-day Mortality, } \\
\%(95 \% \mathrm{Cl})\end{array}$ \\
\hline Number of transports & 10,033 & $10.6(3.6 ; 41.3)$ & $2.1 \%(1.8-2.4 \%)$ \\
\hline Acute cardiac conditions & 1318 & $88.0(53.6 ; 122.3)$ & $4.6 \%(3.4-5.9 \%)$ \\
\hline AMl & 1054 & $91.0(65.0 ; 122.4)$ & $4.0 \%(2.8-5.5 \%)$ \\
\hline STEMI & 340 & $93.0(72.3 ; 120.5)$ & $3.5 \%(1.8-6.2 \%)$ \\
\hline NSTEMI & 714 & $88.4(55.2 ; 124.5)$ & $4.2 \%(2.8-6.1 \%)$ \\
\hline Other acute cardiovascular conditions: & 264 & $65.2(14.2 ; 121.9)$ & $7.0 \%(4.0-11.3 \%)$ \\
\hline UAP & 140 & $62.5(28.6 ; 108.1)$ & $1.8 \%(0.2-6.4 \%)$ \\
\hline Cardiac arrest & 11 & $1.3(1.0 ; 123.3)$ & $60.0 \%(26.2-87.8 \%)$ \\
\hline Ventricular tachycardia & 15 & $73.6(48.6 ; 209.1)$ & $0.0 \%(0.0-30.8 \%)$ \\
\hline Aortic dissection & 21 & $137.4(3.0 ; 266.4)$ & $21.1 \%(6.1-45.6 \%)$ \\
\hline Pulmonary Embolism & 77 & $69.1(4.1 ; 99.8)$ & $4.6 \%(1.0-12.9 \%)$ \\
\hline Other conditions: & 3647 & $16.0(3.9 ; 53.1)$ & $3.6 \%(2.9-4.4 \%)$ \\
\hline Certain infectious and parasitic diseases & 106 & $13.8(2.4 ; 132.3)$ & $11.8 \%(5.6-21.3 \%)$ \\
\hline Neoplasms & 21 & $73.5(3.0 ; 265.2)$ & $35.7 \%(12.8-64.9 \%)$ \\
\hline Endocrine, nutritional and metabolic diseases & 67 & $6.1(3.0 ; 32.7)$ & $8.1 \%(1.7-21.9 \%)$ \\
\hline Mental and behavioral disorders & 108 & $2.7(1.3 ; 15.1)$ & $0.0 \%(0.0-7.0 \%)$ \\
\hline Diseases of the nervous system & 42 & $5.0(2.4 ; 20.5)$ & $3.4 \%(0.1-17.8 \%)$ \\
\hline Diseases of the circulatory system (other than acute) & 1734 & $20.6(7.3 ; 52.3)$ & $2.5 \%(1.7-3.5 \%)$ \\
\hline Diseases of the respiratory system & 599 & $22.3(3.1 ; 93.0)$ & $6.3 \%(4.2-9.0 \%)$ \\
\hline Diseases of the digestive system & 308 & $10.2(2.6 ; 53.7)$ & $2.2 \%(0.7-5.1 \%)$ \\
\hline $\begin{array}{l}\text { Diseases of the musculoskeletal system and } \\
\text { connective tissue }\end{array}$ & 113 & $5.0(2.0 ; 8.9)$ & $2.4 \%(0.3-8.2 \%)$ \\
\hline Diseases of the genitourinary system & 93 & $19.2(5.0 ; 70.3)$ & $3.5 \%(0.4-12.1 \%)$ \\
\hline $\begin{array}{l}\text { Injury, poisoning and certain other consequences } \\
\text { of external causes }\end{array}$ & 152 & $2.9(1.7 ; 6.0)$ & $3.2 \%(0.7-9.1 \%)$ \\
\hline Other diagnoses ${ }^{a}$ & 304 & $10.0(2.8 ; 27.8)$ & $2.4 \%(0.8-5.5 \%)$ \\
\hline No final diagnosis: & 5068 & $7.4(3.0 ; 14.8)$ & $0.4 \%(0.2-0.6 \%)$ \\
\hline Symptoms, signs and abnormal clinical and laboratory findings & 2394 & $3.9(2.0 ; 8.6)$ & $0.5 \%(0.2-0.9 \%)$ \\
\hline $\begin{array}{l}\text { Medical observation and evaluation for suspected diseases } \\
\text { and conditions or other reasons }\end{array}$ & 2674 & $10.3(6.4 ; 19.5)$ & $0.2 \%(0.1-0.6 \%)$ \\
\hline
\end{tabular}

GP General practitioner, UAP Unstable angina pectoris, AMI Acute myocardial infarction, STEMI ST-segment elevation myocardial infarction, NSTEMI non ST-

mortality rate in the overall cohort (6.2\% vs $5.4 \%)$. Mortality in the diagnose-missing group was slightly higher (6.7\%) but as mentioned, this group includes persons dying during ambulance transport or found dead, and subsequently transported to hospital for confirmation of death by a doctor, which can explain the observed higher mortality. We conclude that the risk of selection bias due to exclusion of these patients is very low. There is a risk of immortal time bias in our regression models, since we only include patients surviving to hospital admission. However, in prehospital terminated transports, 30-day mortality among chest pain patients was as low as $3.2 \%$, disputing the possible immortal time bias.
In mortality analysis 70 patients are lost-to-follow-up, meaning that they are registered as inactive in the $\mathrm{Da}$ nish civil registration system. However, only 13 of these where registered as inactive within 30 days after their chest pain event, while the remaining 57 where inactive at the time of their chest pain event (e.g. migrated or without residence in Denmark).

The study is limited by its retrospective design, which affects both the identification of symptoms (if specific information is missing) and potentially biases the effect of patient characteristics, symptoms and comorbidity, since we cannot definitely differentiate between correlation and causality. The identification of chest pain could be limited by the fact that only $37 \%$ of patients 
Table 3 Predictors of mortality and discharge with an acute cardiac diagnosis in all chest pain patients

\begin{tabular}{|c|c|c|c|c|c|c|c|}
\hline & & 30-day mortality & & & Acute cardiac diagn & during in & admission \\
\hline & Total $^{a}$ & Risk Ratio (95\% Cl) & $P$-value & Deaths, n(\%) & Risk Ratio (95\% Cl) & $P$-value & $\begin{array}{l}\text { Acute cardiac } \\
\text { diagnoses, } \mathrm{n}(\%)\end{array}$ \\
\hline Total & 7635 & & & $160(2 \%)$ & & & $1140(15 \%)$ \\
\hline Gender & & & & & & & \\
\hline Female & 3485 & 1.0 (ref.) & & $74(2 \%)$ & 1.0 (ref.) & & $375(11 \%)$ \\
\hline Male & 4150 & $1.06(0.78 ; 1.44)$ & 0.721 & $86(2 \%)$ & $1.77(1.58 ; 1.99)$ & $<0.001$ & 765 (18\%) \\
\hline Age-group & & & & & & & \\
\hline$<30$ & 344 & 1.0 (ref.) & & $2(1 \%)$ & 1.0 (ref.) & & $6(2 \%)$ \\
\hline $30-49$ & 1344 & $0.12(0.01 ; 1.37)$ & 0.089 & $1(\%)$ & $4.71(2.09 ; 10.62)$ & $<0.001$ & $109(8 \%)$ \\
\hline $50-59$ & 1373 & $0.24(0.03 ; 1.68)$ & 0.149 & $2(\%)$ & $9.05(4.05 ; 20.21)$ & $<0.001$ & $208(15 \%)$ \\
\hline $60-69$ & 1691 & $1.94(0.46 ; 8.23)$ & 0.369 & $22(1 \%)$ & $11.77(5.29 ; 26.18)$ & $<0.001$ & $326(19 \%)$ \\
\hline$>=70$ & 2883 & $6.33(1.56 ; 25.61)$ & 0.010 & $133(5 \%)$ & $11.39(5.13 ; 25.32)$ & $<0.001$ & $491(17 \%)$ \\
\hline Acute Myocardial Infarction & MI) & & & & & & \\
\hline No prior AMl & 6785 & 1.0 (ref.) & & $132(2 \%)$ & 1.0 (ref.) & & 1005 (15\%) \\
\hline Prior AMI & 850 & $0.96(0.63 ; 1.46)$ & 0.835 & $28(3 \%)$ & $0.96(0.81 ; 1.14)$ & 0.677 & $135(16 \%)$ \\
\hline Diabetes (DM) & & & & & & & \\
\hline No DM & 6812 & 1.0 (ref.) & & $131(2 \%)$ & 1.0 (ref.) & & $1013(15 \%)$ \\
\hline DM & 823 & $1.11(0.74 ; 1.66)$ & 0.618 & $29(4 \%)$ & $0.97(0.82 ; 1.15)$ & 0.758 & $127(15 \%)$ \\
\hline Congestive heart disease $(C$ & & & & & & & \\
\hline No CHD & 7003 & 1.0 (ref.) & & $128(2 \%)$ & 1.0 (ref.) & & $1065(15 \%)$ \\
\hline $\mathrm{CHD}$ & 632 & $1.33(0.88 ; 2.02)$ & 0.178 & $32(5 \%)$ & $0.69(0.55 ; 0.87)$ & 0.002 & 75 (12\%) \\
\hline Chronic pulmonary disease & PD) & & & & & & \\
\hline No CPD & 6757 & 1.0 (ref.) & & $114(2 \%)$ & 1.0 (ref.) & & $1053(16 \%)$ \\
\hline CPD & 878 & $1.9(1.35 ; 2.68)$ & $<0.001$ & $46(5 \%)$ & $0.59(0.48 ; 0.72)$ & $<0.001$ & $87(10 \%)$ \\
\hline Renal disease (RD) & & & & & & & \\
\hline No or mild RD & 7325 & 1.0 (ref.) & & $139(2 \%)$ & 1.0 (ref.) & & 1097 (15\%) \\
\hline Moderate to severe RD & 310 & $1.7(1.06 ; 2.71)$ & 0.027 & $21(7 \%)$ & $0.9(0.68 ; 1.2)$ & 0.466 & $43(14 \%)$ \\
\hline
\end{tabular}

andividual patients, including only first transport in study period

were triaged using symptom-based dispatch. Instead, symptom-identification relied on automatized text search in notes from electronical prehospital patient records. This method may be sensitive to spelling and abbreviation of words in the records. We sought to compensate for this by including several spellings and known abbreviations of each word, by excluding terms describing rejection of chest pain symptoms and by manual validation of the method in $>5 \%$ of the cohort. The automatized text search showed very good

Table 4 Predictors of mortality and discharge with a acute cardiac diagnosis, according to accompanying symptom in the subgroup of patients with chest pain undergoing criteria-based dispatch

\begin{tabular}{|c|c|c|c|c|c|c|c|}
\hline & \multirow[b]{2}{*}{ Total } & \multicolumn{3}{|l|}{ 30-day mortality } & \multicolumn{3}{|c|}{ Acute cardiac diagnosis during index admission } \\
\hline & & Risk Ratio (95\% Cl) & $P$-value & Deaths, n(\%) & Risk Ratio (95\% Cl) & $P$-value & Acute cardiac diagnoses, $\mathrm{n}(\%)$ \\
\hline Total & 2634 & & & $69(3 \%)$ & & & 397 (15\%) \\
\hline \multicolumn{8}{|l|}{ Accompanying symptoms } \\
\hline Non-responsive & 9 & $9.79(1.31 ; 72.92)$ & 0.026 & $1(11 \%)$ & $2.56(0.74 ; 8.86)$ & 0.136 & $2(22 \%)$ \\
\hline Dyspnoea & 566 & $2.15(1.03 ; 4.51)$ & 0.043 & $26(5 \%)$ & $1.8(1.27 ; 2.56)$ & 0.001 & $82(14 \%)$ \\
\hline Radiation/severe pain $>5$ min & 1068 & $1.25(0.59 ; 2.62)$ & 0.558 & $26(2 \%)$ & $2.06(1.5 ; 2.84)$ & $<0.001$ & $183(17 \%)$ \\
\hline Clammy skin/Uncomfortable/Nausea & 488 & $0.8(0.3 ; 2.12)$ & 0.653 & $7(1 \%)$ & $2.12(1.51 ; 3.0)$ & $<0.001$ & $90(18 \%)$ \\
\hline Other or no & 503 & 1.0 (ref.) & & $9(2 \%)$ & 1.0 (ref.) & & $40(8 \%)$ \\
\hline
\end{tabular}


agreement with the manual reviews $($ kappa $=0.95)$, Additional file 3 .

\section{Discussion}

This retrospective study in a large cohort of patients being transported by ambulance has three major findings. Firstly, chest pain is a very common reason for acute ambulance transport and hospital admission, accounting for one in six emergent ambulance transports. Secondly, acute ambulance transport in chest pain patients is associated with a high degree of overtriage with more than $50 \%$ of transported chest pain patients discharged without any diagnosis of disease and with a very low $(<0.5 \%)$ 30 day-mortality risk. Thirdly, it may be possible to identify a very-low-risk group already at the time of emergency call based on patient characteristics and symptomatology, indicating a potential for downgrading the response in selected chest pain patients.

The rate of chest pain patients transported by ambulance was comparable to the chest pain rate found in recent Swedish and Danish studies, but substantially higher than earlier reported in studies from Norway and the UK [2, 20-23]. The most likely reason for this higher rate is a temporal increase in chest pain cases also reported in other studies [23, 24]. This increase may be explained by an increased focus in the general population and in health care professionals on the need for acute assessment in cases with chest pain. Both chest pain and AMI rates in the present study are also comparable to rates previously reported from European and American ED cohorts [1, 25].

When compared to patients without chest pain, chest pain patients were more often diagnosed with an acute cardiac condition including AMI (13\% vs $2 \%)$, but also more often discharged with no final diagnosis $(51 \%$ vs. $19 \%)$. This is a paradox and reflects on one hand the relevance of chest pain as a symptom of acute cardiovascular disease, but on the other hand also an overtriage of chest pain patients compared to patients presenting with other symptoms. The burden added to the EMS by chest pain patients is exaggerated by the fact that $88 \%$ of these patients were allocated a "grade A" response, thus representing $31 \%$ of all "grade A" responses. The decision to dispatch a "grade A" response is currently not based on an individual risk-estimation, but rather a "no miss" strategy, where the majority of patients with symptoms with even the slightest risk of AMI, are offered the highest level of care. The result is a significant overtriage, where more than half of all chest pain patients are discharged with no disease specific diagnosis and extremely low 30 -day mortality below $0.5 \%$. This advocates the use of a systematic differentiation between high-risk chest pain patients with urgent need of treatment and very low-risk chest pain patients without need for hospital admission. In the EMCC, this may be achieved by including patient characteristics and symptoms (readily accessible at the time of emergency call) in a risk assessment tool.

In explorative analyses on the present cohort (Tables 3 and 4) female gender, younger age, chronic pulmonary disease, absence of dyspnoea, absence of radiation/severe pain for $>5 \mathrm{~min}$ and absence of clammy skin/uncomfortable/nausea were associated with being discharged without an acute cardiac diagnosis and surviving 30 days after a chest pain event. Applied in a diagnostic model for the composite endpoint of no acute cardiac diagnosis during index admission and surviving 30 days from chest pain event, Model 2 presented acceptable sensitivity, NPV and negative likelihood ratio. However, since these data were retrospective, they were not gathered with this in mind. Thus, though risk assessment using patient characteristics and clinical presentation/ accompanying symptoms seems promising, safety of such a strategy should be further refined and explored in prospective studies. Future studies should aim at confirming that an estimated very low risk justifies a lower prehospital dispatch priority and potentially prehospital rule out of AMI and other acute conditions already at medical emergency call.

Clinical implementation of a risk assessment strategy may further unfold as the patient pass through the EMS system. Early rule-out of AMI in the ED using patient characteristics and clinical presentation in combination with high-sensitive troponin has been validated in several recent studies [26-28]. Even earlier rule-out of AMI could be achieved by combining high-sensitive troponin with copeptin, maybe even in the prehospital setting, when equipment for point-of-care copeptin measurement is developed [29-31]. The prerequisites for this dual marker approach are examined in an ongoing randomized controlled study [32]. With a risk assessment tool used at time of emergency call, resource downgrading after ambulance dispatch may be based on incorporation of subsequent clinical assessment, ECG-findings and biomarker results in a stepwise, individual risk assessment model, even before reaching hospital.

\section{Conclusion}

Chest pain is the reason for $16 \%$ of all ambulance transports. AMI is only diagnosed in $11 \%$ of chest pain patients. The mortality in chest pain patients is lower than in other patients transported to hospital by ambulance, and particularly low in chest pain patients not receiving a diagnosis of disease during index admission. Structured risk assessment using patient characteristics and symptoms seems to hold a potential for early resource downgrading in low risk chest pain patients, already in the prehospital setting. 


\section{Additional files}

Additional file 1: Patient flowchart. Flowchart of study patients.
(PDF $121 \mathrm{~kb}$ )

Additional file 2: Prehospitally terminated transports and transports missing symptoms or final diagnosis. Characteristics, symptoms and diagnoses in transports terminated prehospitally, or in which either symptoms or final diagnosis is missing. (PDF $424 \mathrm{~kb}$ )

Additional file 3: Identification of chest pain. Details on Danish Index codes indicating chest pain and the automatic text search and validation of this. (PDF $605 \mathrm{~kb}$ )

Additional file 4: ICD-10 codes. ICD-10 diagnoses used to categorize final diagnosis, calculate Charlson Comorbidity Index (CCI), and to identify known comorbidity. (PDF 531 kb)

Additional file 5: Data for Fig. 1. Raw values and categories (Fig. 1a) and time-to-event-or-censoring and censor status per patient (Fig. 1b) (XLS $7940 \mathrm{~kb}$ )

\section{Abbreviations}

AMl: Acute myocardial infarction; CCl: Charlson Comorbidity Index; CCU: Coronary Care Unit; Cl : Confidence interval; ECG: Electrocardiogram; ED: Emergency Department; EMCC: Emergency Medical Communication Centres; EMS: Emergency Medical Service; GP: General Practitioner; IQR: Interquartile ranges; LOS: Length of stay; NPV: Negative predictive value

\section{Acknowledgements}

The authors thank Ingunn Skogstad Riddervold, PhD, for providing vital assistance in acquiring the prehospital data for this publication. Further, the authors thank Kamilla Pernille Bjerre, MD; Anne Dybro, MD; Kasper Korsholm, MD; Mikkel Giehm-Reese, MD; Sivagowry Rasalingam Mørk, MD; and Anne Sofie Frederiksen, MD for their high valued assistance in validating the text-search algorithm.

\section{Authors' contributions}

CKP, MTB, CS, HMS, KKD and CJT conceived the study. CKP and KF were responsible for data collection and managed the data. MTB, CS, CJT, MHS and KKD supervised the conduct of the study. CJT, CS and MTB provided statistical advice on data analysis. CKP analysed the data. CKP drafted this manuscript, and all authors read and approved the final manuscript.

\section{Funding}

This research did not receive any specific grant from funding agencies in the public, commercial, or not-for-profit sectors.

\section{Availability of data and materials}

The datasets generated and/or analysed during the current study are not publicly available owing to protection of personal data, but data and statistical codes are available from the corresponding author on reasonable request and with permission of relevant Danish authorities.

\section{Ethics approval and consent to participate}

The study was approved by the Danish Data Protection Agency (1-16-02788-17) and the National Board of Health (3-3013-2321/1). According to Danish law, informed consent and ethics committee approval are not required in observational studies.

\section{Consent for publication}

Not applicable.

\section{Competing interests}

CKP: Roche Diagnostics: honorarium.

KKD: No conflicts of interest.

HMS: No conflicts of interest.

CJT: No conflicts of interest.

CS: Roche Diagnostics: honorarium.

KF: No conflicts of interest.

MTB: No conflicts of interest.

\section{Author details}

${ }^{1}$ Department of Cardiology, Aarhus University Hospital, Palle Juul-Jensens Boulevard 99, 8200 Aarhus N, Denmark. '2Department of Anesthesiology, Aarhus University Hospital, Aarhus, Denmark. ${ }^{3}$ Research and Development, Prehospital Emergency Medical Services, Central Denmark Region, Aarhus, Denmark. ${ }^{4}$ Department of Internal Medicine, Regional Hospital Horsens, Horsens, Denmark. ${ }^{5}$ Department of Cardiology, Regional Hospital Viborg, Viborg, Denmark.

Received: 8 July 2019 Accepted: 14 August 2019

Published online: 29 August 2019

\section{References}

1. Mockel M, Searle J, Muller R, et al. Chief complaints in medical emergencies: do they relate to underlying disease and outcome? The Charite emergency medicine study (CHARITEM). Eur J Emerg Med. 2013;20(2):103-8. https://doi. org/10.1097/MEJ.0b013e328351e609.

2. Burman RA, Zakariassen E, Hunskaar S. Acute chest pain - a prospective population based study of contacts to Norwegian emergency medical communication centres. BMC Emerg Med. 2011;11:9. https://doi.org/10.11 86/1471-227X-11-9.

3. Niska R, Bhuiya F, Xu J. National Hospital Ambulatory Medical Care Survey: 2007 Emergency department summary. Natl Health Stat Report. 2010;26:1-31.

4. Rui PKK. In: Statistics NCfH, editor. National Hospital Ambulatory Medical Care Survey: 2015 Emergency Department Summary Table; 2015.

5. Forberg JL, Henriksen LS, Edenbrandt L, et al. Direct hospital costs of chest pain patients attending the emergency department: a retrospective study. BMC Emerg Med. 2006;6:6. https://doi.org/10.1186/1471-227X-6-6.

6. Lassen JF, Botker HE, Terkelsen CJ. Timely and optimal treatment of patients with STEMI. Nat Rev Cardiol. 2013;10(1):41-8. https://doi.org/10.1038/ nrcardio.2012.156.

7. Terkelsen CJ, Sorensen JT, Maeng M, et al. System delay and mortality among patients with STEMI treated with primary percutaneous coronary intervention. JAMA. 2010;304(7):763-71. https://doi.org/10.1001/jama.201 0.1139 .

8. Karlson BW, Herlitz J, Pettersson $\mathrm{P}$, et al. Patients admitted to the emergency room with symptoms indicative of acute myocardial infarction. J Intern Med. 1991;230(3):251-8.

9. Kohn MA, Kwan E, Gupta M, et al. Prevalence of acute myocardial infarction and other serious diagnoses in patients presenting to an urban emergency department with chest pain. J Emerg Med. 2005;29(4):383-90. https://doi. org/10.1016/j.jemermed.2005.04.010.

10. Gupta M, Tabas JA, Kohn MA. Presenting complaint among patients with myocardial infarction who present to an urban, public hospital emergency department. Ann Emerg Med. 2002;40(2):180-6.

11. Moller TP, Ersboll AK, Tolstrup JS, et al. Why and when citizens call for emergency help: an observational study of 211,193 medical emergency calls. Scand J Trauma Resusc Emerg Med. 2015;23:88. https://doi.org/10.11 86/s13049-015-0169-0.

12. Herlitz J, Bang A, Isaksson L, et al. Ambulance despatchers' estimation of intensity of pain and presence of associated symptoms in relation to outcome in patients who call for an ambulance because of acute chest pain. Eur Heart J. 1995;16(12):1789-94.

13. Regions D. The REGIONS - in BRIEF. In: Regions D, editor. WEBSITE, 2011.

14. Botker MT, Terkelsen CJ, Sorensen JN, et al. Long-term mortality of emergency medical services patients. Ann Emerg Med. 2017;70(3):366-73 e3. https://doi.org/10.1016/j.annemergmed.2016.12.017.

15. Regions D. Dansk Index for Akuthjælp 1.5. 2004.

16. Lynge E, Sandegaard JL, Rebolj M. The Danish National Patient Register. Scand J Public Health. 2011;39(7 Suppl):30-3. https://doi.org/10.1177/14034 94811401482

17. Schmidt M, Pedersen L, Sorensen HT. The Danish civil registration system as a tool in epidemiology. Eur J Epidemiol. 2014;29(8):541-9. https://doi.org/1 0.1007/s10654-014-9930-3.

18. Schmidt M, Schmidt SA, Sandegaard JL, et al. The Danish National Patient Registry: a review of content, data quality, and research potential. Clin Epidemiol. 2015;7:449-90. https://doi.org/10.2147/CLEP.S91125.

19. Thygesen SK, Christiansen CF, Christensen S, et al. The predictive value of ICD-10 diagnostic coding used to assess Charlson comorbidity index conditions in the population-based Danish National Registry of patients. 
BMC Med Res Methodol. 2011;11:83. https://doi.org/10.1186/1471-22 88-11-83.

20. Sovso MB, Klojgaard TA, Hansen PA, et al. Repeated ambulance use is associated with chronic diseases - a population-based historic cohort study of patients' symptoms and diagnoses. Scand I Trauma Resusc Emerg Med. 2019;27(1):46. https://doi.org/10.1186/s13049-019-0624-4.

21. Rawshani A, Larsson A, Gelang C, et al. Characteristics and outcome among patients who dial for the EMS due to chest pain. Int J Cardiol. 2014;176(3): 859-65. https://doi.org/10.1016/j.jijcard.2014.08.004.

22. Goodacre S, Cross E, Arnold J, et al. The health care burden of acute chest pain. Heart. 2005;91 (2):229-30. https://doi.org/10.1136/hrt.2003.027599.

23. McDevitt-Petrovic O, Kirby K, Shevlin M. The prevalence of non-cardiac chest pain (NCCP) using emergency department (ED) data: a Northern Ireland based study. BMC Health Serv Res. 2017;17(1):549. https://doi.org/1 0.1186/s12913-017-2493-8.

24. Thang ND, Karlson BW, Bergman B, et al. Patients admitted to hospital with chest pain--changes in a 20-year perspective. Int J Cardiol. 2013;166(1):1416. https://doi.org/10.1016/j.ijcard.2011.10.014.

25. Bhuiya FA, Pitts SR, LF MC. Emergency department visits for chest pain and abdominal pain: United States, 1999-2008. NCHS Data Brief. 2010;43:1-8.

26. Mueller C, Giannitsis E, Christ M, et al. Multicenter evaluation of a 0-hour/1hour algorithm in the diagnosis of myocardial infarction with highsensitivity cardiac troponin T. Ann Emerg Med. 2016. https://doi.org/10.1 016/j.annemergmed.2015.11.013.

27. Body R, Burrows G, Carley S, et al. High-sensitivity cardiac troponin t concentrations below the limit of detection to exclude acute myocardia infarction: a prospective evaluation. Clin Chem. 2015;61(7):983-9. https://doi. org/10.1373/dinchem.2014.231530.

28. Body R, Mueller C, Giannitsis E, et al. The use of very low concentrations of high sensitivity troponin $\mathrm{T}$ to rule out acute myocardial infarction using a single blood test. Acad Emerg Med. 2016. https://doi.org/10.1111/acem.13 012.

29. Mockel M, Searle J, Hamm C, et al. Early discharge using single cardiac troponin and copeptin testing in patients with suspected acute coronary syndrome (ACS): a randomized, controlled clinical process study. Eur Heart J. 2015;36(6):369-76. https://doi.org/10.1093/eurheartj/ehu178.

30. Stengaard C, Sorensen JT, Ladefoged SA, et al. The potential of optimizing prehospital triage of patients with suspected acute myocardial infarction using high-sensitivity cardiac troponin T and copeptin. Biomarkers. 2017; 22(3-4):351-60. https://doi.org/10.1080/1354750X.2016.1265008.

31. Stengaard C, Sorensen JT, Rasmussen MB, et al. Prehospital diagnosis of patients with acute myocardial infarction. Diagnosis (Berl). 2016;3(4):155-66. https://doi.org/10.1515/dx-2016-0021.

32. Pedersen CK, Stengaard C, Sondergaard $\mathrm{H}$, et al. A multicentre, randomized, controlled open-label trial to compare an accelerated rule-out protocol using combined prehospital copeptin and in-hospital high sensitive troponin with standard rule-out in patients suspected of acute myocardial infarction - the AROMI trial. Trials. 2018;19(1):683. https://doi.org/10.1186/s13 063-018-2990-z.

\section{Publisher's Note}

Springer Nature remains neutral with regard to jurisdictional claims in published maps and institutional affiliations.

Ready to submit your research? Choose BMC and benefit from:
- fast, convenient online submission
- thorough peer review by experienced researchers in your field
- rapid publication on acceptance
- support for research data, including large and complex data types
- gold Open Access which fosters wider collaboration and increased citations
- maximum visibility for your research: over 100M website views per year
At BMC, research is always in progress.
Learn more biomedcentral.com/submissions

